\title{
Polymer-Magnetic Composite Fibers for Remote-Controlled Drug Release
}

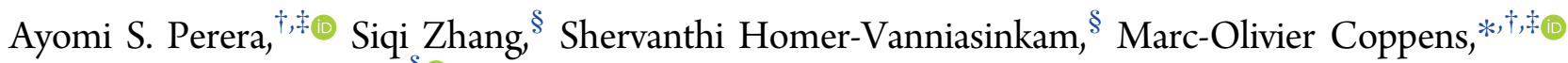 \\ and Mohan Edirisinghe*, ${ }^{*} \odot$
}

${ }^{\dagger}$ Centre for Nature Inspired Engineering, ${ }^{\ddagger}$ Department of Chemical Engineering, and ${ }^{\S}$ Department of Mechanical Engineering, University College London, Torrington Place, London WC1E 7JE, U.K.

\section{Supporting Information}

\begin{abstract}
An efficient method is reported, for the fabrication of composite microfibers that can be magnetically actuated and are biocompatible, targeting controlled drug release. Aqueous solutions of polyvinyl alcohol, incorporated with citric acid-coated $\mathrm{Fe}_{3} \mathrm{O}_{4}$ magnetic nanoparticles (MNPs), are subject to infusion gyration to generate 100$300 \mathrm{~nm}$ diameter composite fibers, with controllable MNP loading. The fibers are stable in polar solvents, such as ethanol, and do not show any leaching of MNPs for over 4 weeks. Using acetaminophen as an example, we demonstrate that this material is effective in immobilization and triggered release of drugs, which is achieved by a moving external magnetic field. The remote actuation ability, coupled with biocompatibility and lightweight property, renders enormous potential for these fibers to be used as a smart drug release agent.
\end{abstract}

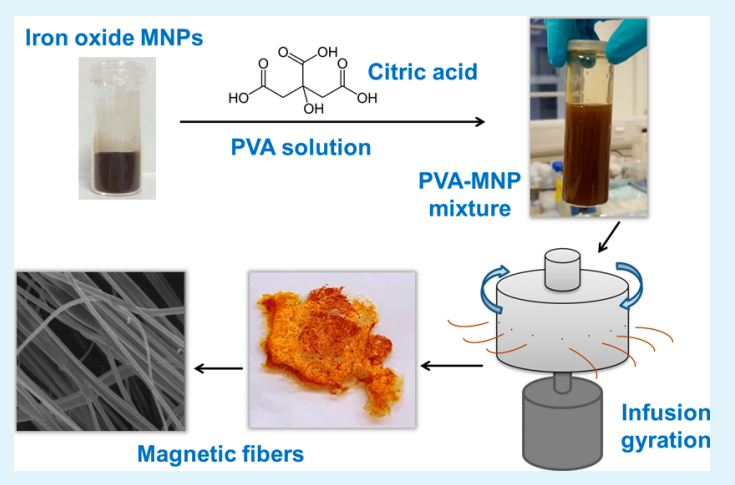

KEYWORDS: nanofibers, nanomaterials, polymer-magnetic composites, magnetic actuation, controlled drug release, infusion gyration

\section{INTRODUCTION}

Magnetically triggered release of active pharmaceutical ingredients is a rapidly growing area of research with applications targeted toward efficient, minimally invasive pathways of drug delivery. ${ }^{1-4}$ Such studies usually involve the use of superparamagnetic nanoparticles, which are either functionalized as drug-carriers ${ }^{5,6}$ or incorporated with polymers to produce composites in the form of membranes, ${ }^{7}$ liposomes, ${ }^{8-10}$ protein microspheres, ${ }^{3}$ gels, ${ }^{11}$ etc. These materials have become highly significant in the advancement of diagnosis and treatment of cancer and cardiovascular diseases, in addition to leading toward breakthroughs in regenerative medicine. ${ }^{12-16}$ The magnetic component is typically activated via hyperthermia or chemical signals to trigger the release of the drug. ${ }^{17,18}$ Actuation of the drug-carrying platform by an external magnetic field (i.e., magnetic actuation), however, remains scarcely explored but has enormous potential in biomedicine. ${ }^{19,20}$ Such systems can potentially lead to remotecontrolled, precise, and safer pathways of drug delivery and also pave the way for advances in the rapidly evolving field of microrobotics and for applications in medicine. ${ }^{20-22}$

Recent reports indicate that in vitro drug release, in particular, can be improved using conventional carrier systems such as mesoporous silica, in combination with magnetic nanoparticles (MNPs) for triggered cargo release. ${ }^{23-25}$ Such systems have great potential for the treatment of cancer and other related diseases. ${ }^{26}$ These systems, however, need physical (e.g., thermal or light) or chemical (e.g., $\mathrm{pH}$ or redox changes) stimuli or enzymatic catalysis to initiate the release action, which require either invasive procedures or are limited in efficiency and controllability. In contrast, systems containing MNPs give rapid responses, with greater noninvasiveness, and can be easily controlled by external magnetic fields. MNPs also have the advantage of being easily integrated with organic components such as polymers to create composite materials with novel and improved capabilities. ${ }^{12}$

Polymer-based nanofibers have been investigated for a myriad of biomedical applications, including scaffolds for tissue engineering, materials for wound dressing, and vehicles for drug delivery. ${ }^{27}$ Nanoscale fibers are of particular interest in biomedicine, as their morphology resembles biological tissue. They also have high surface area and surface energy and can be organized into porous hierarchical structures, which are highly desirable properties for cell and tissue adhesion as well as the adsorption of drug molecules. The physical structure of the fibers can be readily customized to adapt to different applications. In such instances, synthesis is mostly achieved via electrospinning. ${ }^{28-30}$ This technique has been explored extensively to achieve hollow core-shell microparticleencapsulated fibers. ${ }^{31}$ Furthermore, materials used for fiber formation have been developed to facilitate the incorporation of fibers with biodegradable ${ }^{32,33}$ and antibiotic properties ${ }^{34}$ and even those that include living tissue. ${ }^{35-37}$ Extensive progress

Received: March 23, 2018

Accepted: April 12, 2018

Published: April 12, 2018 
has also been made in the scale-up of such materials targeting industrial applications. $^{38}$

Despite the wealth of research conducted, such nanofibers have not yet, however, made it to the clinical trial stage of biomedical applications. This can be attributed to multiple factors, from mass production to long-term stability of the fibers in vivo. For example, electrospinning, which is the main synthesis method of fiber formation, poses many challenges to their sustained usage in medical applications. The lack of control over fiber diameter and pore sizes, as well as the random nonwoven nature of the fibers produced, causes difficulty in cell penetration, which is a key factor in sustained use of scaffolds for tissue engineering. ${ }^{39,40}$ Although electrospinning is effective in producing microscale fibers, smaller diameters are difficult to obtain. ${ }^{41}$ Attempts at customizing fiber morphology via this technique are complicated and lead to lower yields. ${ }^{42}$ Moreover, electrospun fibers have inherently weaker mechanical strength than cast fibers, and the solvents and cross-linking agents involved in the process often lead to toxicity and noncompatibility in biological systems. ${ }^{43}$

Alternative spinning techniques have been developed to counteract some of the drawbacks of electrospinning. ${ }^{44}$ These, however, are not without flaws themselves. Biospinning, for example, is a technique suitable for producing fibers with greater mechanical strength, such as scaffolds for tendons or bones. ${ }^{45}$ Nevertheless, this method is hampered by high cost, difficulty in scale-up, longer production times, and lack of customizability. Melt spinning can create fibers by extruding a heated polymer through a spinneret with textural control for cell applications. ${ }^{46,47}$ Yet, high energy costs, expensive equipment, and difficulty in producing cell-incorporating fibers are considerable impediments. The latter issue can be solved via an interfacial complexation process, to encapsulate cells, which is also cheaper; ${ }^{48}$ despite this, limitations in scale-up and material and dimension control are significant disadvantages. Overall, a technique to produce such biocompatible fibers in a facile, fast, and cost-effective manner, with controllable sizes and the potential for scale-up, is highly desirable. If the above is also coupled with the ability of remote actuation, it can lead to significant advancements in drug delivery and tissue engineering.

The goal of this study is to demonstrate a magnetically actuated drug delivery system, based on polyvinyl alcohol (PVA)-MNP fibers, generated via infusion gyration. The fibers are made of biocompatible components in order to target biomedical applications, specifically drug release. The ability of these fibers to be actuated via an external magnetic field is demonstrated, along with extensive characterization of their physicochemical properties. The release of controlled quantities of acetaminophen, via magnetic actuation of this material, is explored. Furthermore, the advantages and potential scope of application, beyond drug delivery, are discussed.

\section{EXPERIMENTAL SECTION}

All reagents and chemicals were used as received, without further modification.

2.1. Preparation of PVA and PVA-MNP Solutions. Three types of PVA polymer powder (Sigma-Aldrich, Gillingham, UK), with different molecular weights, were taken in the following weight ratios to obtain three viscous solutions (Table 1): (1) PVA (363 170, 87$89 \%$ hydrolyzed), (2) PVA (363 138, 98-99\% hydrolyzed), (3) PVA ( $363065,99+\%$ hydrolyzed). The three solutions were made by mixing in the relevant weight of each type of PVA powder with
Table 1. Molecular Weights and Weight Ratios of PVA Polymers Used for Pure PVA and PVA-MNP Fiber Fabrication

\begin{tabular}{clc} 
PVA type & molecular weight & weight \% \\
1 & $13000-23000$ & 22 \\
2 & $31000-50000$ & 10 \\
3 & $146000-186000$ & 7 \\
\hline
\end{tabular}

distilled water and heating under magnetic stirring at $90^{\circ} \mathrm{C}$, for $5 \mathrm{~h}$, to achieve homogeneous mixtures.

Next, the three solutions were mixed in a volume ratio of $1: 1: 1$, to make a total of $30 \mathrm{~mL}$ and magnetically stirred at ambient temperature $\left(23^{\circ} \mathrm{C}\right)$ for $24 \mathrm{~h}$. This mixture was stored in sealed glass vials until use. All fiber samples used in this study consisted of the above mixture. Subsequently, this solution was mixed with $5 \%(\mathrm{w} / \mathrm{w}) \mathrm{Fe}_{3} \mathrm{O}_{4}$ magnetic nanopowder $(\sim 20 \mathrm{~nm})$, coated with citric acid (see section 2.2 below), and vigorously vortexed for $5 \mathrm{~min}$ to obtain PVA-MNP solutions. Both the pure PVA and PVA-MNP solutions were then spun into fibers, according to the procedures described in section 2.3.

2.2. Citric Acid Coating of MNPs. MNPs were subject to acid coating prior to mixing with PVA, in order to ensure effective dispersion in the aqueous polymer solution. Citric acid (SigmaAldrich, ACS grade $>99.8 \%$ ) was dissolved in deionized water in a ratio of $0.5 \mathrm{mg} / \mathrm{mL}$, at $90{ }^{\circ} \mathrm{C}$ for $1 \mathrm{~h}$, under magnetic stirring. Then, the stirrer was removed, and a relevant weight of $\mathrm{Fe}_{3} \mathrm{O}_{4}$ MNPs (US Research Nanomaterials, Inc., $20 \mathrm{~nm}$ ) was added to the solution and mixed for another $1 \mathrm{~h}$. Afterward, the acid-coated MNPs were precipitated by placing a magnet under the bottom of the vessel, and the solution was decanted. The collected MNPs were then washed twice, with $100 \mathrm{~mL}$ of deionized water, and dried at $80^{\circ} \mathrm{C}$ in an oven, for $3 \mathrm{~h}$ or until a constant weight was obtained.

2.3. Fabrication of Fibers. The fibers were fabricated using a previously reported ${ }^{49}$ in-house built experimental setup, using the principle of infusion gyration (Figure 1; also see Supporting

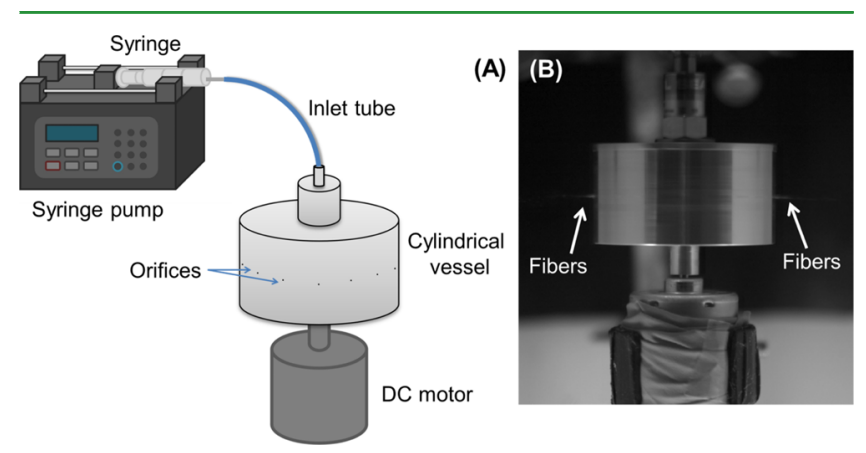

Figure 1. Making polymer-based fibers via infusion gyration. (A) Schematic diagram of the spinning process. (B) High-speed camera image of the spinning cylinder showing fiber formation.

Information Video S1). The device consists of a hollow aluminum rotary vessel, $25 \mathrm{~mm}$ in height and $60 \mathrm{~mm}$ in diameter, and contains 20 small round orifices. Each orifice is of $0.5 \mathrm{~mm}$ in diameter and lies along the central circumference of the cylindrical vessel. The vessel is connected to and powered by a dc motor and a speed controller, which can provide variable speeds of rotation. The rotational speed was fixed at $36000 \mathrm{rpm}$ for this study. The top of the vessel contains a removable cap, with a rotary joint connected to a lead. The other tip of the lead is connected to a syringe pump (PHD 4400 Programmable Syringe Pump, Harvard Apparatus). The flow rate of the solution to the vessel is tuned via the syringe pump and was held at $4 \mathrm{~mL} / \mathrm{min}$ during this study. A stationary steel mesh was placed $120 \mathrm{~mm}$ away from the rotating vessel to help collect the fibers. The solutions consisting of either PVA or MNPs containing PVA were spun at ambient conditions of $23{ }^{\circ} \mathrm{C}$ and $\sim 40 \%$ relative humidity. After the 
spinning was completed, all samples were transferred to sealed containers for further characterization.

2.4. Characterization of PVA and PVA-MNP Fibers. Fiber samples were observed under a Nikon Eclipse ME 600 optical microscope, fitted with a MicroPublisher 3.3 RTV, 3.3 megapixel CCD Color-Bayer Mosaic, Real Time Viewing camera (Media Cybernetics, Marlow, UK). Size and morphology of the fibers were analyzed using field emission scanning electron microscopy (FESEM) with a JEOL JSM6310F instrument. All samples were coated with carbon prior to imaging, using a Quorum K975X turbo-pumped thermal evaporator (Quorum Technologies Ltd., East Sussex, UK). Chemical characterization was achieved via FTIR spectroscopy, with a Bruker VERTEX 70 instrument. An INCA X-sight EDAX system (Oxford Instruments) was used with Hitachi S-3400N for EDX (energy-dispersive X-ray) spectroscopy for surface elemental analysis. Stability of the composite fibers was studied by immersing in an absolute ethanol solution for 4 weeks, followed by testing for $\mathrm{Fe}_{3} \mathrm{O}_{4}$ leaching via UV-vis spectroscopy, using an Agilent Technologies Cary 4000 UV-vis spectrophotometer.

2.5. Magnetic Actuation and Characterization. Magnetic actuation of the MNP-containing fibers was achieved using commercial neodymium magnets (emagnets UK, EP336, $20 \mathrm{~mm}$ dia $\times 5 \mathrm{~mm}, \mathrm{~N} 42$, NiCuNi plated, $1.4 \mathrm{~T}$ ), in air and in ethanol. The fibers effectively responded to the external magnetic field while contained in glass vials open to air or in glass vials containing absolute ethanol. The fibers could also easily be guided in a channel containing ethanol. Magnetization measurements were performed using a Quantum Design MPMS SQUID-VSM magnetometer. Samples were weighed and mounted within polycarbonate holders and subsequently measured $\left( \pm 1 \mathrm{~T}, 796 \mathrm{kA} \mathrm{m}^{-1}\right.$, at $\left.300 \mathrm{~K}\right)$.

2.6. Drug Release Experiments. Drug release experiments were conducted in absolute ethanol medium, with acetaminophen (SigmaAldrich, analytical standard) as a model drug. For each experiment, 5.0 $\mathrm{mg}$ of acetaminophen was transferred on to $60.0 \mathrm{mg}$ of magnetic fibers. The fibers were wetted with three drops of absolute ethanol, prior to transfer of acetaminophen on to them. In order to ensure that the drug was effectively immobilized in the fiber network, the acetaminophen powder was spread throughout and pressed against the fibers with a spatula. This system was then transferred to a glass vial containing $10.0 \mathrm{~mL}$ of absolute ethanol. Both control experiments and actuation experiments were conducted in duplicate, to measure the release of acetaminophen into the solution with time. For the actuation experiments, the fiber-drug system was continuously moved via four stacked external magnets (see section 2.5). Samples of $500 \mu \mathrm{L}$ of the ethanol solute were taken out from the system, first after every 1 $\mathrm{min}$, up to $5 \mathrm{~min}$, and then at every $5 \mathrm{~min}$, for a total period of $30 \mathrm{~min}$. Subsequently, the samples were analyzed via UV-vis spectrophotometry, to determine the concentration of acetaminophen released via magnetic actuation. The $500 \mu \mathrm{L}$ taken out for sampling was replaced with new ethanol each time, in order to keep a total constant volume of $10 \mathrm{~mL}$ of ethanol throughout the experiment. The same procedure was followed to conduct control experiments, with the exception of the magnetic actuation.

The concentration of acetaminophen released into the solution was measured via ultraviolet-visible (UV-vis) spectrophotometry at a characteristic absorbance wavelength of $248 \mathrm{~nm}$. UV-vis spectroscopy was carried out using the absorbance mode of a BioTek Synergy H1 multidetection reader. These measurements were compared to a calibration curve, and the acetaminophen concentrations of the experimental samples were determined by the Beer-Lambert law.

\section{RESULTS AND DISCUSSION}

3.1. PVA and PVA-MNP Fibers via Infusion Gyration. Spinning of polymer solutions through micro-orifices under high pressure and speed is a technique recently optimized to mass-produce nanofibers. ${ }^{49,50}$ This technology was extended to include nanoparticles ${ }^{51}$ and protein ${ }^{52}$-containing polymer solutions to generate composite fibers. In the latter, ${ }^{52}$ the flow rate, rather than pressure, was controlled during gyration; this is called infusion gyration. Here, this method was used to successfully generate fibers consisting of PVA polymer and PVA-MNP.

The morphology of the fibers was studied using optical and scanning electron microscopies. Optical microscopy revealed a fiber matrix containing small amounts of beads distributed in the matrix, for both pure PVA (Supporting Information, Figure S1A) and PVA-MNP (Figure 2A,B) samples. These beads

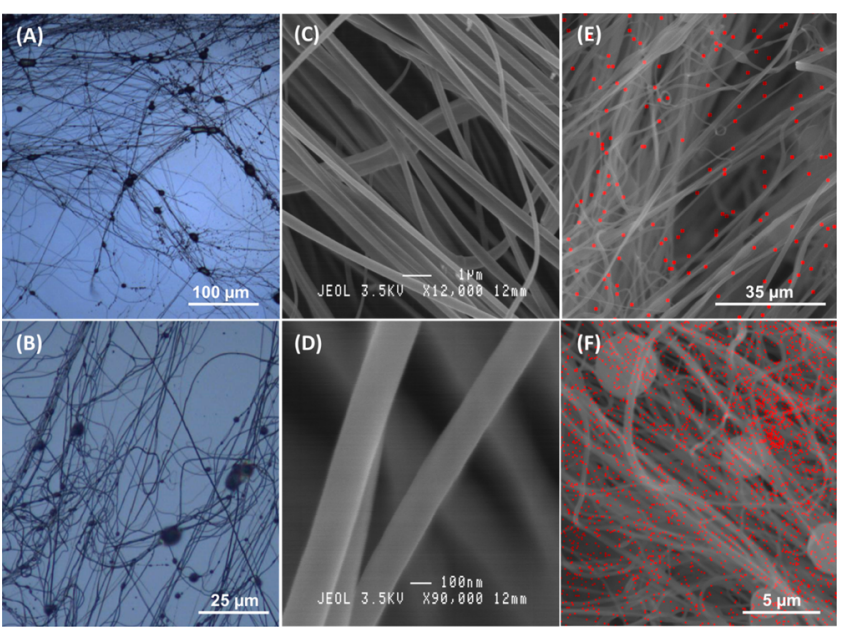

Figure 2. PVA-MNP fibers $(5 \%(w / w))$. (A,B) Optical microscopy images, (C,D) SEM images, and (E,F) SEM dot mapping: the red dots indicate the presence of $\mathrm{Fe}$ in fibers.

were observed to be hollow structures via SEM and could arise from a combination of factors, such as the rheological properties of the polymer solution together with the spinning speed and pressure. ${ }^{53}$ Fibers made from both PVA and PVAMNP appeared to have a distribution of $100-300 \mathrm{~nm}$ in diameter (Figure 2C,D and Supporting Information, Figure $\mathrm{S} 1 \mathrm{~B})$. The incorporation of $\mathrm{Fe}_{3} \mathrm{O}_{4}$ MNPs into the fibers was visualized via $\mathrm{EDX}$ dot mapping, which revealed that the iron oxide powder was distributed homogeneously on the fibers (Figure 2E,F).

Chemical composition of the fibers was analyzed using FTIR and EDX. Examination of the FTIR peaks allowed the identification of all typical peaks for PVA and citric acid (Figure 3A). These include the broad peaks at $3300 \mathrm{~cm}^{-1}$ from the stretching mode of inter- and intramolecular hydrogen bonds of $\mathrm{O}-\mathrm{H} ; 2920$ and $2850 \mathrm{~cm}^{-1}$ from the $\mathrm{C}-\mathrm{H}$ vibrational mode of alkyl groups; $1735 \mathrm{~cm}^{-1}$ from the stretching mode of $\mathrm{C}=\mathrm{O}$ of the carbonyl groups; $1085 \mathrm{~cm}^{-1}$ from the $\mathrm{C}-\mathrm{O}-\mathrm{C}$ stretching mode; a shoulder at $\sim 1141 \mathrm{~cm}^{-1}$ from $\mathrm{C}-\mathrm{O}$ stretching of crystalline PVA; and $1242 \mathrm{~cm}^{-1}$ from the symmetric stretching mode of citric acid. ${ }^{54-56}$

However, the characteristic vibrational bands of $\mathrm{Fe}_{3} \mathrm{O}_{4}$ usually found at 634,582 , and $397 \mathrm{~cm}^{-1}$, characteristic of magnetite, ${ }^{57}$ were not discernible via the instrument used. The presence of surface Fe was successfully detected using EDX and was found to be in the range of 5-10\% of atomic weight for different samples (Figure 3B,C).

In order to correctly quantify the amount of MNPs loaded on to the fibers, the material was subject to superconducting quantum interference device (SQUID) analysis. It was found that the fibers were composed of $4.9 \%$ magnetic component by weight (due to $\mathrm{Fe}_{3} \mathrm{O}_{4} \mathrm{MNPs}$ ), as determined by the fraction of the mass magnetization for the PVA-MNP fibers $(M=2.6 \mathrm{~A}$ 

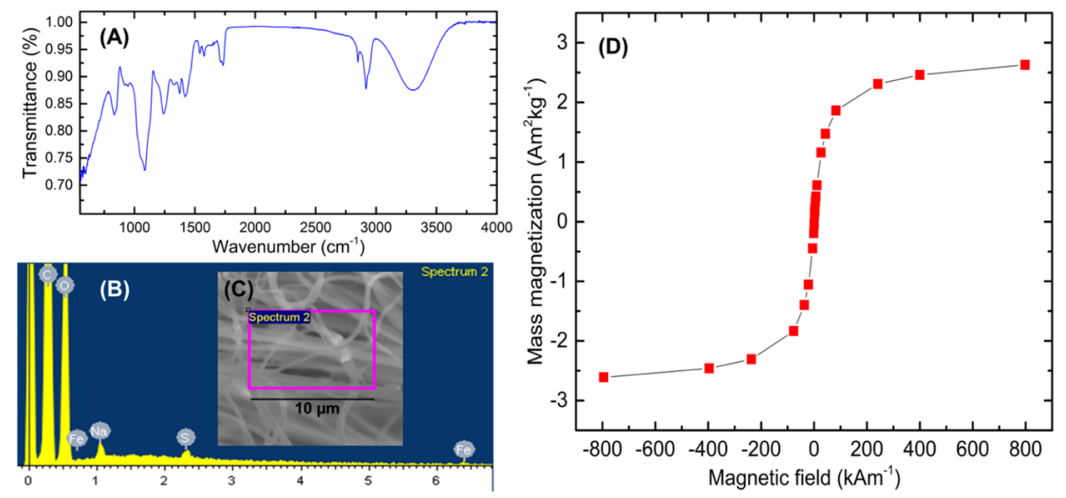

Figure 3. Characterization of chemical composition and magnetic content of the PVA-MNP fibers. (A) FTIR spectrum, (B) elemental analysis using EDX, (C) area of fiber sample subject to EDX analysis, and (D) mass magnetization behavior of the MNP-PVA fiber sample.

$\left.\mathrm{m}^{2} \mathrm{~kg}^{-1}\right)$ and the pure MNPs $\left(M=53.8 \mathrm{~A} \mathrm{~m}^{2} \mathrm{~kg}^{-1}\right.$ ) (Figure S3 and Figure 3D). Because the polymer solution fed to the spinning apparatus consisted of $5 \%$ of MNPs by weight, this shows that the process of infused gyration is highly effective in generating fibers with minimal loss of the magnetic material. This observation, coupled with the previously investigated efficacy of the gyration process, ${ }^{52}$ renders potential for scaled up production of magnetic fibers using this technique.

3.2. Controlled Release of Acetaminophen via Magnetic Actuation of PVA-MNP Fibers. The ability to release controlled quantities of drugs via application of an external stimulus onto the carrier platform is a highly advantageous feature for drug delivery. This was successfully demonstrated by the controlled release of acetaminophen loaded on the PVA-MNP fibers, via an external magnetic field. Absolute ethanol was chosen as the medium for these experiments, in which the magnetic fibers were stable. UVvis experiments conducted on the supernatant of PVA-MNP fibers stored in absolute ethanol for 4 weeks showed no traces of $\mathrm{Fe}_{3} \mathrm{O}_{4}$ leaching (Supporting Information Figure S2). Acetaminophen was chosen as a model drug for this study due to its high solubility in ethanol ${ }^{58}$ and its characteristic UVvis absorption. ${ }^{59}$ This drug was effectively immobilized onto the PVA-MNP fibers (Figure 4A), following which the drug-fiber system was transferred to an absolute ethanol medium and studied for the release of acetaminophen with time, both with and without magnetic actuation (Figure 4B,C and Supporting Information Videos S2 and S3). Separate experiments were conducted in duplicate, for the control (i.e., without actuation) and actuated studies. Samples were taken out at relevant time intervals and subject to UV-vis analysis. The ethanol volume was kept constant for all samples throughout the study by replacing the sampling volume.

Acetaminophen has strong UV absorption with a prominent peak at $243 \mathrm{~nm}$ and a broad shoulder at around $290 \mathrm{~nm}$, in water. ${ }^{60}$ In absolute ethanol, these peaks were observed at 248 and $295 \mathrm{~nm}$, respectively (Figure 5A). A calibration curve for acetaminophen was obtained using standard solutions and was used to determine the concentration of its quantities released during the control and actuation experiments of the acetaminophen-loaded fiber system. On the basis of the above results, the concentration and the cumulative \% of acetaminophen released were plotted against time (Figures S4 and $5 \mathrm{~B}$, respectively).

It was evident that the magnetically actuated fiber system released significantly more acetaminophen with time, compared

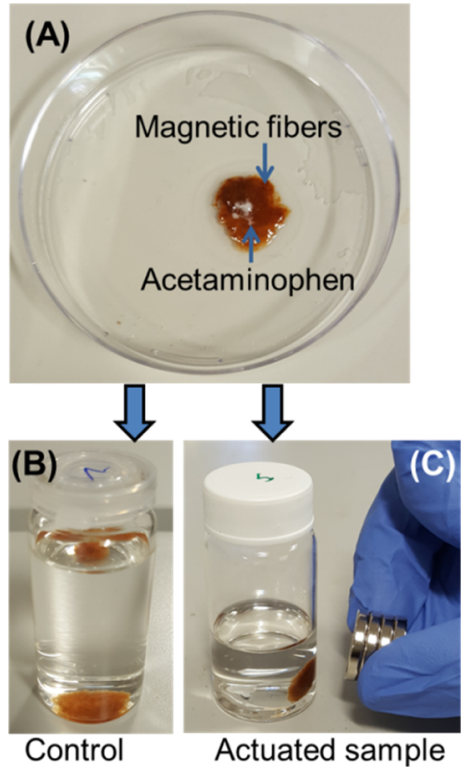

Figure 4. Drug release experiments using magnetic fibers. (A) Loading of acetaminophen onto the fibers, (B) control experiment without any actuation, and (C) fiber-drug system actuated via an external magnet.

to the nonactuated system. For the actuated samples, a rapid release of acetaminophen was observed during the first $5 \mathrm{~min}$, followed by a gradual increase, up to $15 \mathrm{~min}$, following which the release plateaued until the end of the experiment, at $30 \mathrm{~min}$. This behavior is consistent with a "burst-release" mechanism. ${ }^{61}$ About $50 \%$ of the drug was released within $2-3$ min and over $90 \%$ by the end of $15 \mathrm{~min}$ of actuation. No statistically significant further increase in release concentration is observed over the 15-30 min time frame. This may be because further drug release is restricted due to the strong adsorption or possible entrapment of remaining drug within the fiber matrix. It is also noteworthy that, because the drug release is triggered by movement (i.e., magnetic actuation), we are unable to ensure that the acetaminophen concentration is homogeneous throughout the test solution. This may result in greater differences in duplicate experiments conducted for actuated samples compared to the controls, bringing about larger experimental errors for the former. In contrast, the control samples released significantly lower amounts of drug during the first $5 \mathrm{~min}$, followed by a slower gradual increase in drug release over the next $20 \mathrm{~min}$, and finally showed a slight decrease during the last $5 \mathrm{~min}$. This behavior can be attributed to simple 

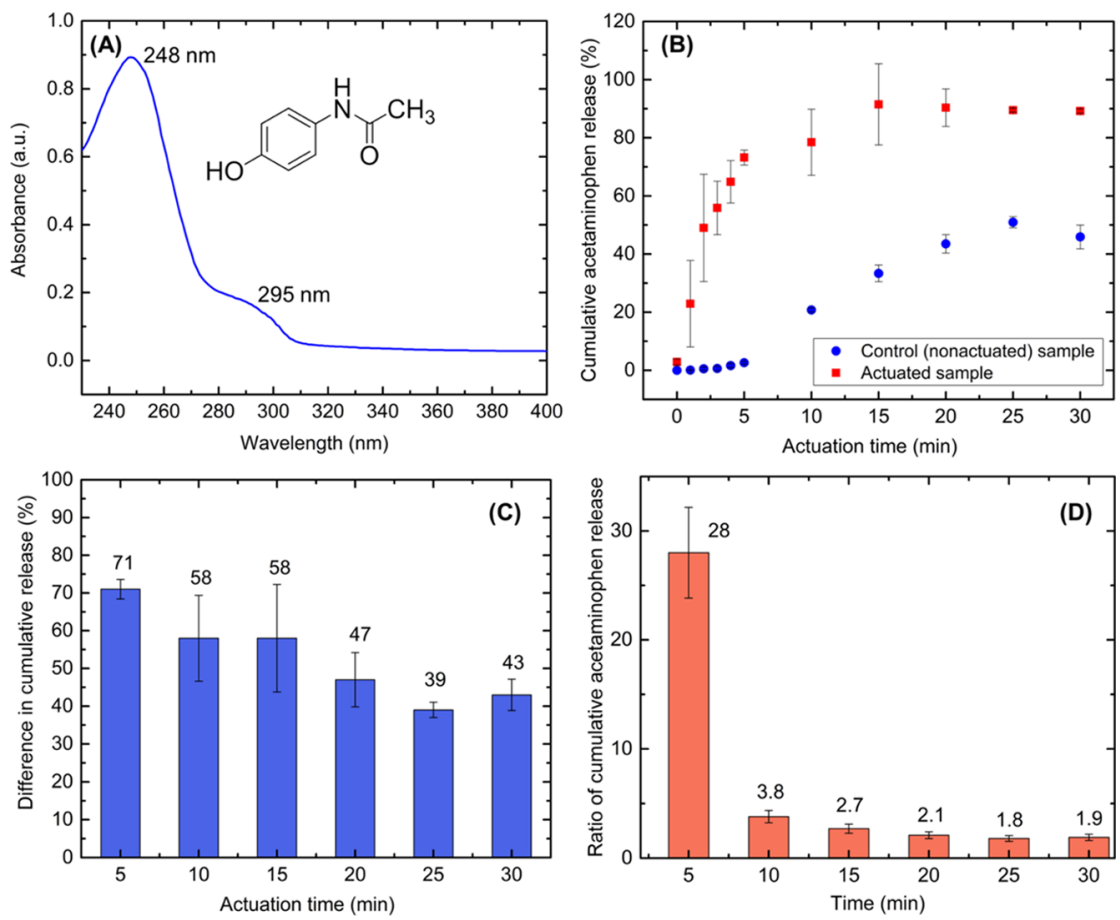

Figure 5. Use of magnetic fibers for controlled release of acetaminophen with and without magnetic actuation. (A) Chemical structure and UV-vis absorption spectrum of acetaminophen, (B) cumulative weight percentages of acetaminophen released with time. Here, the control experiment represents the equivalent release of acetaminophen without magnetic (or any other type of) actuation. (C) Effect of magnetic actuation on drug release with time: the difference between actuated and nonactuated cumulative release curves. (D) Ratio of acetaminophen release from actuated and nonactuated fibers.

diffusion of the immobilized acetaminophen from the fiber network.

The difference between the cumulative \% of acetaminophen released over time from the actuated and nonactuated fibers, for the 5-30 min time frame, is depicted in Figure 5C. By the end of the first $5 \mathrm{~min}$, there is a stark difference of about $71 \%$ of acetaminophen release between the two types of samples. This amounts to approximately 28 times higher drug release from actuated fibers compared to a nonactuated sample, as depicted by the ratios in Figure 5D. This is followed by steadily decreasing ratios of release up to $15 \mathrm{~min}$, and by the end of the 30 min study period, about twice as much acetaminophen continues to release via magnetic actuation, compared to diffusion. The above is clear evidence that remote magnetic actuation can bring about significant changes in both an absolute and a relative sense, for the release of drugs from PVA-MNP fibers.

On the basis of the above results, it is evident that the PVAMNP fibers are capable of fast drug release during smaller time scales and slower, consistent drug release over time, via magnetic actuation. This novel, biocompatible, cost-effective, and controllable technique has the potential to be used clinically, as an effective tool for triggered drug release. Application of this mode of drug delivery is particularly attractive in wound care due to several reasons: (1) it can be used in the community by tissue viability nurses, (2) repeated drug delivery can be effected, without necessarily changing or removing the dressings, and (3) chemical debridement of wounds (as opposed to surgical debridement for which one needs an anesthetic, theater time, and, often, a physician to conduct the procedure) can be carried out if the relevant drug is incorporated into the PVA-MNP fibers. Moreover, there is increasingly a focus to move the management of wound care into the community, which achieves two important NHS objectives: (a) it relieves hospital bed pressures and (b) patients can be managed in the convenience of their own homes and are not subjected to the challenges of being an inpatient.

This technology also has potential applications for "sitespecific" treatment due to its customizable properties. For example, the sensitivity of magnetic actuation of the fibers, namely, the minimum depth and distance from the magnetic source needed for actuation, can be improved by increasing either the MNP concentration or the power of the magnet. Then, the material can possibly be applied in treatment of atherosclerotic plaque in the carotid artery or, in simpler terms, for prevention of a stroke. In this instance, the magnetic fibers could be administered intravenously, and then the magnetic actuation could be directed to the carotid artery in the neck so that the drug would be released only at this specific site. If deeper penetration of the magnetic force can be achieved safely, then one could envisage widening the portfolio of use within the body in a number of conditions, for example, acute conditions such as sepsis and chronic diseases, such as inflammation in arthritis and inflammatory bowel disease, and in a range of oncological therapies.

\section{CONCLUSIONS}

PVA nanofibers incorporating $5 \%(\mathrm{w} / \mathrm{w})$ of magnetic $\mathrm{Fe}_{3} \mathrm{O}_{4}$ nanoparticles were successfully and reproducibly prepared by infusion gyration. The fibers were shown to be stable when stored in ethanol, without any leaching of MNPs. The magnetic particles impart sufficient magnetization to the fibers to support actuation via external magnets. Acetaminophen was used to successfully demonstrate controlled drug release using the PVA-MNP fibers, when subject to magnetic actuation, 
bringing about over $90 \%$ cumulative release in $15 \mathrm{~min}$. Compared to nonactuated controls, the magnetic fibers showed approximately $71 \%$ more release of the drug within $5 \mathrm{~min}$, in absolute terms, and nearly 28 times higher release, in relative terms. This is promising for applications of this technology as an efficient remote-controlled method for drug delivery. The above, coupled together with facile, cost-effective material synthesis, with proven ability to scale up, offers attractive opportunities in clinical application of the magnetic fibers.

\section{ASSOCIATED CONTENT}

\section{S Supporting Information}

The Supporting Information is available free of charge on the ACS Publications website at DOI: 10.1021/acsami.8b04774.

SEM images of pure PVA fibers; MNP leaching studies for PVA-MNP fibers conducted via UV-vis absorbance of supernatant solutions; mass magnetization behavior of both pure $\mathrm{MNP}\left(M=2.6 \mathrm{~A} \mathrm{~m}^{2} \mathrm{~kg}^{-1}\right)$ and $5 \% \mathrm{MNP}-$ PVA fiber $\left(M=53.8 \mathrm{~A} \mathrm{~m}^{2} \mathrm{~kg}^{-1}\right)$ samples; and concentration of acetaminophen released with time (PDF)

High-speed camera video showing fiber formation during the infusion gyration process (ZIP)

Procedure for magnetic actuation of fibers loaded with acetaminophen (ZIP)

Transportation of the magnetic fibers along a tube using magnetic actuation, demonstrating the scope of actuation (ZIP)

\section{AUTHOR INFORMATION}

\section{Corresponding Authors}

*E-mail: m.coppens@ucl.ac.uk (M.-O.C.).

*E-mail: m.edirisinghe@ucl.ac.uk (M.E.).

ORCID

Ayomi S. Perera: 0000-0003-2139-040X

Marc-Olivier Coppens: 0000-0002-1810-2537

Mohan Edirisinghe: 0000-0001-8258-7914

\section{Author Contributions}

A.S.P. and S.Z. made equal contributions to this work. The manuscript was written through contributions of all authors. All authors have given approval to the final version of the manuscript.

\section{Funding}

The Engineering and Physical Sciences Research Council (UK) is gratefully acknowledged for support of gyratory forming work (grants EP/L023059/1 and EP/N034228/1) and for a "Frontier Engineering" award, EP/K038656/1.

\section{Notes}

The authors declare no competing financial interest.

\section{ACKNOWLEDGMENTS}

The authors gratefully acknowledge the valuable contributions from Justin Siefker and Michele Lynch of the Department of Chemical Engineering, UCL, for assistance with FTIR and UV-vis experiments, Dr. Paul Southern of the UCL Healthcare Biomagnetics Laboratory for SQUID analysis, and Dr. Tom Gregory of the UCL Institute of Archaeology for SEM and EDX.

\section{ABBREVIATIONS}

PVA, polyvinyl alcohol; MNPs, magnetic nanoparticles; dc, direct current; EDX, energy-dispersive X-ray; $M_{\mathrm{w}}$, molecular weight; UV-vis, ultraviolet-visible; SQUID, superconducting quantum interference device; FESEM, field emission scanning electron microscope; FTIR, Fourier transform infrared; NHS, National Health Service (UK)

\section{REFERENCES}

(1) Ulbrich, K.; Holá, K.; Šubr, V.; Bakandritsos, A.; Tuček, J.; Zbořil, R. Targeted Drug Delivery with Polymers and Magnetic Nanoparticles: Covalent and Noncovalent Approaches, Release Control, and Clinical Studies. Chem. Rev. 2016, 116, 5338-5431.

(2) Wang, Y.; Kohane, D. S. External triggering and triggered targeting strategies for drug delivery. Nat. Rev. Mater. 2017, 2, 17020.

(3) Chandna, A.; Batra, D.; Kakar, S.; Singh, R. A review on target drug delivery: magnetic microspheres. J. Acute Dis. 2013, 2, 189-195.

(4) Arruebo, M.; Fernández-Pacheco, R.; Ibarra, M. R.; Santamaría, J. Magnetic nanoparticles for drug delivery. Nano Today 2007, 2, 22-32.

(5) Jurgons, R.; Seliger, C.; Hilpert, A.; Trahms, L.; Odenbach, S.; Alexiou, C. Drug loaded magnetic nanoparticles for cancer therapy. J. Phys.: Condens. Matter 2006, 18, S2893.

(6) Perera, A. S. Sustainable Magnetic Nanocatalysts in Heterogeneous Catalysis. Magnetic Nanomaterials: Applications in Catalysis and Life Sciences; Royal Society of Chemistry, 2017; Chapter 4, pp 99-119.

(7) Hoare, T.; Timko, B. P.; Santamaria, J.; Goya, G. F.; Irusta, S.; Lau, S.; Stefanescu, C. F.; Lin, D.; Langer, R.; Kohane, D. S. Magnetically Triggered Nanocomposite Membranes: A Versatile Platform for Triggered Drug Release. Nano Lett. 2011, 11, 13951400.

(8) Oliveira, H.; Pérez-Andrés, E.; Thevenot, J.; Sandre, O.; Berra, E.; Lecommandoux, S. Magnetic field triggered drug release from polymersomes for cancer therapeutics. J. Controlled Release 2013, $169,165-170$.

(9) Lee, J.-H.; Ivkov, R.; Blumenthal, R. Magnetically Triggered Drug Release from Liposome Embedded Gel. J. Nanomed. Biother. Discovery 2014, 4, 130-136.

(10) Bi, H.; Ma, S.; Li, Q.; Han, X. Magnetically triggered drug release from biocompatible microcapsules for potential cancer therapeutics. J. Mater. Chem. B 2016, 4, 3269-3277.

(11) Hoare, T.; Timko, B. P.; Santamaria, J.; Goya, G. F.; Irusta, S.; Lau, S.; Stefanescu, C. F.; Lin, D.; Langer, R.; Kohane, D. S. Magnetically-triggered Nanocomposite Membranes: a Versatile Platform for Triggered Drug Release. Nano Lett. 2011, 11, 1395-1400.

(12) Veiseh, O.; Gunn, J. W.; Zhang, M. Design and fabrication of magnetic nanoparticles for targeted drug delivery and imaging. Adv. Drug Delivery Rev. 2010, 62, 284-304.

(13) Gobbo, O. L.; Sjaastad, K.; Radomski, M. W.; Volkov, Y.; PrinaMello, A. Magnetic Nanoparticles in Cancer Theranostics. Theranostics 2015, 5, 1249-1263.

(14) Sun, C.; Lee, J.; Zhang, M. Magnetic Nanoparticles in MR Imaging and Drug Delivery. Adv. Drug Delivery Rev. 2008, 60, 12521265.

(15) Qureshi, A.; Gurbuz, Y.; Niazi, J. H. Biosensors for cardiac biomarkers detection: A review. Sens. Actuators, B 2012, 171, 62-76.

(16) Gao, Y.; Lim, J.; Teoh, S.-H.; Xu, C. Emerging translational research on magnetic nanoparticles for regenerative medicine. Chem. Soc. Rev. 2015, 44, 6306-6329.

(17) Hergt, R.; Dutz, S.; Müller, R.; Zeisberger, M. Magnetic particle hyperthermia: nanoparticle magnetism and materials development for cancer therapy. J. Phys.: Condens. Matter 2006, 18, S2919.

(18) Pankhurst, Q. A.; Thanh, N. T. K.; Jones, S. K.; Dobson, J. Progress in applications of magnetic nanoparticles in biomedicine. J. Phys. D: Appl. Phys. 2009, 42, 224001.

(19) Fusco, S.; Huang, H.-W.; Peyer, K. E.; Peters, C.; Häberli, M.; Ulbers, A.; Spyrogianni, A.; Pellicer, E.; Sort, J.; Pratsinis, S. E.; Nelson, B. J.; Sakar, M. S.; Pané, S. Shape-Switching Microrobots for Medical 
Applications: The Influence of Shape in Drug Delivery and Locomotion. ACS Appl. Mater. Interfaces 2015, 7, 6803-6811.

(20) Temel, F. Z.; Yesilyurt, S. Magnetically actuated micro swimming of bio-inspired robots in mini channels. 2011 IEEE International Conference on Mechatronics, 13-15 April 2011, 2011; Vol. 2011, pp 342-347.

(21) Xu, T.; Yu, J.; Yan, X.; Choi, H.; Zhang, L. Magnetic Actuation Based Motion Control for Microrobots: An Overview. Micromachines 2015, 6, 1346-1364.

(22) Floyd, S.; Pawashe, C.; Sitti, M. An untethered magnetically actuated micro-robot capable of motion on arbitrary surfaces. 2008 IEEE International Conference on Robotics and Automation, 19-23 May 2008, 2008; Vol. 2008, pp 419-424.

(23) Thomas, C. R.; Ferris, D. P.; Lee, J.-H.; Choi, E.; Cho, M. H.; Kim, E. S.; Stoddart, J. F.; Shin, J.-S.; Cheon, J.; Zink, J. I. Noninvasive Remote-Controlled Release of Drug Molecules in Vitro Using Magnetic Actuation of Mechanized Nanoparticles. J. Am. Chem. Soc. 2010, 132, 10623-10625.

(24) Singh, R. K.; Patel, K. D.; Kim, J.-J.; Kim, T.-H.; Kim, J.-H.; Shin, U. S.; Lee, E.-J.; Knowles, J. C.; Kim, H.-W. Multifunctional Hybrid Nanocarrier: Magnetic CNTs Ensheathed with Mesoporous Silica for Drug Delivery and Imaging System. ACS Appl. Mater. Interfaces 2014, 6, 2201-2208.

(25) Chen, J.-P.; Yang, P.-C.; Ma, Y.-H.; Tu, S.-J.; Lu, Y.-J. Targeted delivery of tissue plasminogen activator by binding to silica-coated magnetic nanoparticle. Int. J. Nanomed. 2012, 7, 5137-5149.

(26) Mody, V. V.; Cox, A.; Shah, S.; Singh, A.; Bevins, W.; Parihar, H. Magnetic nanoparticle drug delivery systems for targeting tumor. Appl. Nanosci. 2014, 4, 385-392.

(27) Leung, V.; Ko, F. Biomedical applications of nanofibers. Polym. Adv. Technol. 2011, 22, 350-365.

(28) Suwantong, O. Biomedical applications of electrospun polycaprolactone fiber mats. Polym. Adv. Technol. 2016, 27, 12641273.

(29) Liu, H.; Ding, X.; Zhou, G.; Li, P.; Wei, X.; Fan, Y. Electrospinning of nanofibers for tissue engineering applications. $J$. Nanomater. 2013, 2013, 1-11.

(30) Rodríguez, K.; Gatenholm, P.; Renneckar, S. Electrospinning cellulosic nanofibers for biomedical applications: structure and in vitro biocompatibility. Cellulose 2012, 19, 1583-1598.

(31) Agarwal, S.; Wendorff, J. H.; Greiner, A. Use of electrospinning technique for biomedical applications. Polymer 2008, 49, 5603-5621.

(32) Ashammakhi, N.; Ndreu, A.; Piras, A. M.; Nikkola, L.; Sindelar, T.; Jukola, H.; Harlin, A.; Gomes, M. E.; Neves, N. M.; Chiellini, E.; Chiellini, F.; Hasirci, V.; Redl, H.; Reis, R. L. Biodegradable nanomats produced by electrospinning: Expanding multifunctionality and potential for tissue engineering. J. Nanosci. Nanotechnol. 2007, 7, $862-882$.

(33) Teo, W.-E.; He, W.; Ramakrishna, S. Electrospun scaffold tailored for tissue-specific extracellular matrix. Biotechnol. J. 2006, 1, 918-929.

(34) Hipler, U.-C.; Elsner, P.; Fluhr, J. W. Antifungal and antibacterial properties of a silver-loaded cellulosic fiber. J. Biomed. Mater. Res., Part B 2006, 77, 156-163.

(35) Townsend-Nicholson, A.; Jayasinghe, S. N. Cell Electrospinning: a Unique Biotechnique for Encapsulating Living Organisms for Generating Active Biological Microthreads/Scaffolds. Biomacromolecules 2006, 7, 3364-3369.

(36) Jayasinghe, S. N.; Irvine, S.; McEwan, J. R. Cell electrospinning highly concentrated cellular suspensions containing primary living organisms into cell-bearing threads and scaffolds. Nanomedicine 2007, 2, 555-567.

(37) Yan, S.; Li, X.; Dai, J.; Wang, Y.; Wang, B.; Lu, Y.; Shi, J.; Huang, P.; Gong, J.; Yao, Y. Electrospinning of PVA/sericin nanofiber and the effect on epithelial-mesenchymal transition of A549 cells. Mater. Sci. Eng., C 2017, 79, 436-444.

(38) Persano, L.; Camposeo, A.; Tekmen, C.; Pisignano, D. Industrial Upscaling of Electrospinning and Applications of Polymer Nanofibers: A Review. Macromol. Mater. Eng. 2013, 298, 504-520.
(39) Guimarães, A.; Martins, A.; Pinho, E. D.; Faria, S.; Reis, R. L.; Neves, N. M. Solving cell infiltration limitations of electrospun nanofiber meshes for tissue engineering applications. Nanomedicine 2010, 5, 539-554.

(40) Martins, A.; Araújo, J. V.; Reis, R. L.; Neves, N. M. Electrospun nanostructured scaffolds for tissue engineering applications. Nanomedicine 2007, 2, 929-942.

(41) Zhang, Y.; Lim, C. T.; Ramakrishna, S.; Huang, Z.-M. Recent development of polymer nanofibers for biomedical and biotechnological applications. J. Mater. Sci.: Mater. Med. 2005, 16, 933-946.

(42) Zafar, M.; Najeeb, S.; Khurshid, Z.; Vazirzadeh, M.; Zohaib, S.; Najeeb, B.; Sefat, F. Potential of Electrospun Nanofibers for Biomedical and Dental Applications. Materials 2016, 9, 73.

(43) Tamimi, E.; Ardila, D. C.; Haskett, D. G.; Doetschman, T.; Slepian, M. J.; Kellar, R. S.; Vande Geest, J. P. Biomechanical Comparison of Glutaraldehyde-Crosslinked Gelatin Fibrinogen Electrospun Scaffolds to Porcine Coronary Arteries. J. Biomech. Eng. 2015, 138, 011001.

(44) Tamayol, A.; Akbari, M.; Annabi, N.; Paul, A.; Khademhosseini, A.; Juncker, D. Fiber-based tissue engineering: Progress, challenges, and opportunities. Biotechnol. Adv. 2013, 31, 669-687.

(45) Gosline, J. M.; Guerette, P. A.; Ortlepp, C. S.; Savage, K. N. The mechanical design of spider silks: from fibroin sequence to mechanical function. J. Exp. Biol. 1999, 202, 3295-3303.

(46) Gomes, M. E.; Azevedo, H. S.; Moreira, A. R.; Ellä, V.; Kellomäki, M.; Reis, R. L. Starch-poly ( $\varepsilon$-caprolactone) and starchpoly(lactic acid) fibre-mesh scaffolds for bone tissue engineering applications: structure, mechanical properties and degradation behaviour. J. Tissue Eng. Regener. Med. 2008, 2, 243-252.

(47) Sinclair, K. D.; Webb, K.; Brown, P. J. The effect of various denier capillary channel polymer fibers on the alignment of NHDF cells and type I collagen. J. Biomed. Mater. Res., Part A 2010, 95, 11941202.

(48) Wan, A. C. A.; Liao, I.-C.; Yim, E. K. F.; Leong, K. W. Mechanism of Fiber Formation by Interfacial Polyelectrolyte Complexation. Macromolecules 2004, 37, 7019-7025.

(49) Mahalingam, S.; Edirisinghe, M. Forming of Polymer Nanofibers by a Pressurised Gyration Process. Macromol. Rapid Commun. 2013, 34, 1134-1139.

(50) Mahalingam, S.; Raimi-Abraham, B. T.; Craig, D. Q. M.; Edirisinghe, M. Solubility-spinnability map and model for the preparation of fibres of polyethylene (terephthalate) using gyration and pressure. Chem. Eng. J. 2015, 280, 344-353.

(51) Illangakoon, U. E.; Mahalingam, S.; Wang, K.; Cheong, Y.-K.; Canales, E.; Ren, G. G.; Cloutman-Green, E.; Edirisinghe, M.; Ciric, L. Gyrospun antimicrobial nanoparticle loaded fibrous polymeric filters. Mater. Sci. Eng., C 2017, 74, 315-324.

(52) Zhang, S.; Karaca, B. T.; VanOosten, S. K.; Yuca, E.; Mahalingam, S.; Edirisinghe, M.; Tamerler, C. Coupling Infusion and Gyration for the Nanoscale Assembly of Functional Polymer Nanofibers Integrated with Genetically Engineered Proteins. Macromol. Rapid Commun. 2015, 36, 1322-1328.

(53) Hong, X.; Edirisinghe, M.; Mahalingam, S. Beads, beaded-fibres and fibres: Tailoring the morphology of poly(caprolactone) using pressurised gyration. Mater. Sci. Eng., C 2016, 69, 1373-1382.

(54) Mallapragada, S. K.; Peppas, N. A. Dissolution mechanism of semicrystalline poly(vinyl alcohol) in water. J. Polym. Sci., Part B: Polym. Phys. 1996, 34, 1339-1346.

(55) Mansur, H. S.; Sadahira, C. M.; Souza, A. N.; Mansur, A. A. P. FTIR spectroscopy characterization of poly (vinyl alcohol) hydrogel with different hydrolysis degree and chemically crosslinked with glutaraldehyde. Mater. Sci. Eng., C 2008, 28, 539-548.

(56) Bichara, L. C.; Lanús, H. E.; Ferrer, E. G.; Gramajo, M. B.; Brandán, S. A. Vibrational Study and Force Field of the Citric Acid Dimer Based on the SQM Methodology. Adv. Phys. Chem. 2011, 2011, $1-10$.

(57) Cornell, R. M.; Schwertmann, U. The Iron Oxides: Structure, Properties, Reactions, Occurrences and Uses; Wiley, 2006. 
(58) Granberg, R. A.; Rasmuson, Å. C. Solubility of Paracetamol in Pure Solvents. J. Chem. Eng. Data 1999, 44, 1391-1395.

(59) Glavanović, S.; Glavanović, M.; Tomišić, V. Simultaneous quantitative determination of paracetamol and tramadol in tablet formulation using UV spectrophotometry and chemometric methods. Spectrochim. Acta, Part A 2016, 157, 258-264.

(60) Behera, S.; Ghanty, S.; Ahmad, F.; Santra, S.; Banerjee, S. UVVisible Spectrophotometric Method Development and Validation of Assay of Paracetamol Tablet Formulation. J. Anal. Bioanal. Tech. 2012, $3,151-157$.

(61) Huang, X.; Brazel, C. S. On the importance and mechanisms of burst release in matrix-controlled drug delivery systems. J. Controlled Release 2001, 73, 121-136. 